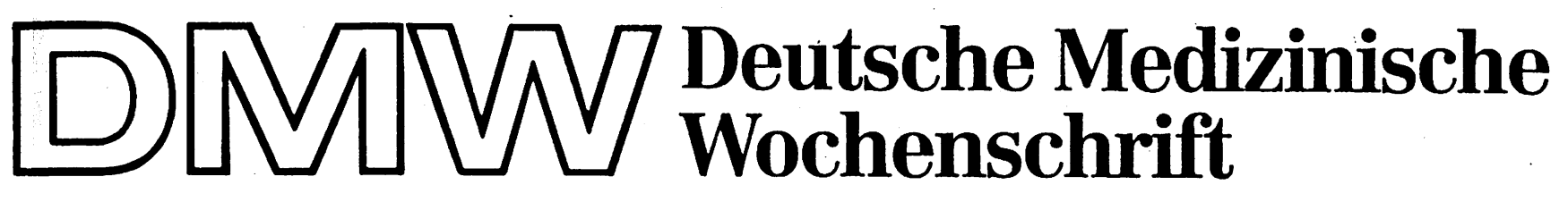

Anschrift für Schriftleitung und Georg Thieme Verlag: 7000 Stuttgart 30, Rüdigerstraße 14

Schriftleitung:

Dr.med.habil. R. Augustin stuttgart Prof.Dr.W. Siegenthaler zürich

Prof. Dr. M. Rothmund Marburg Prof. Dr. J. R. Siewert München Prof. Dr. P. C. Scriba München Prof. Dr. A. Sturm Herne/Bochum

Register für den 117. Jahrgang 1. Halbjahr 1992 Hefte 1-26 


\title{
Riesenzellarteriitis mit isoliertem Befall der Femoralarterien
}

\author{
C. Stautner-Brückmann, C. Haschka, G. Baumann, J. Bogner und F. A. Spengel \\ Medizinische Poliklinik der Universität München (Vorstand: Prof. Dr. N. Zöllner) und Gefäßchirurgische Abteilung \\ (Chefarzt: Prof. Dr. G. Baumann) des Krankenhauses München-Schwabing
}

\begin{abstract}
Eine 48jährige bis dahin gesunde Frau litt an über mehrere Monate zunehmenden Schmerzen in der Hüfte, der Hals- und Lendenwirbelsäule sowie im Schultergürtel. Zudem trat eine Claudicatio intermittens nach 100-200 m Gehstrecke auf. Die Blutsenkungsgeschwindigkeit lag bei $35-43 \mathrm{~mm}$ in der 1 . Stunde; die IgM-Konzentration war auf $610 \mathrm{mg} / \mathrm{dl}$ erhöht. Angiographisch zeigten sich symmetrische Verschlüsse der A. femoralis superficialis und der A. profunda femoris beiderseits. Da eine ultrahochdosierte Kurzlyse mit Streptokinase erfolglos blieb, wurden Gewebeproben aus der Femoralarterie entnommen. Histologisch war eine floride Arteriitis sichtbar, teilweise als Riesenzellarteriitis mit Zerstörung elastischer Strukturen. Die angrenzende Muskulatur war unauffällig. Eine anschließend durchgeführte Biopsie der A. temporalis erbrachte Normalbefunde. Die Patientin wurde zunächst für 4 Wochen mit $60 \mathrm{mg} / \mathrm{d}$ Methylprednisolon sowie mit $100 \mathrm{mg} / \mathrm{d}$ Acetylsalicylsäure behandelt. Daraufhin ging die Blutsenkungsgeschwindigkeit zurück, die schmerzfreie Gehstrecke nahm zu. Auch nach stufenweiser Reduktion der Steroiddosis bis zum Absetzen blieb die Patientin bis heute beschwerdefrei.
\end{abstract}

\section{Giant-cell arteritis limited to the femoral arteries}

A previously healthy 48-year-old woman developed, over several months, increasing pain in her hips, cervical and lumbar spine as well as the shoulders. In addition she developed intermittent claudication after walking $100-200 \mathrm{~m}$. Erythrocyte sedimentation rate (ESR) was 35-43 $\mathrm{mm}$ in the first hour; the IgM concentration was raised to $610 \mathrm{mg} / \mathrm{dl}$. Angiography demonstrated symmetrical occlusion of both superficial femoral arteries. An attempt at thrombolysis having failed, a biopsy of the femoral artery wall was obtained. This revealed a florid arteritis, in part as giant-cell arteritis with destruction of the elastic structures, while the adjacent muscle was unchanged. Subsequent biopsy of the temporal artery was normal. Treatment consisted at first of $60 \mathrm{mg}$ prednisolone and $100 \mathrm{mg}$ aspirin, both daily for 4 weeks. The ESR fell and the free-of-pain walking distance rose. There has been no recurrence of pain after stepwise reduction over one year of the steroid dosage until its discontinuation.
Die Riesenzellarteriitis, auch Polymyalgia arteriitica bzw. rheumatica oder Arteriitis temporalis genannt, geht mit einer Vaskulitis vorwiegend der supraaortalen Äste einher und tritt überwiegend im Alter über 50 Jahren auf. Frauen sind häufiger betroffen als Männer. Meist beginnt die Erkrankung mit allgemeinem Krankheitsgefühl und Myalgien im Schulter- sowie Beckenbereich. Typischerweise ist die Blutsenkungsgeschwindigkeit im akuten Stadium auf mehr als $50 \mathrm{~mm}$ in der 1 . Stunde beschleunigt; die Fraktion der $\alpha_{2}$-Globuline in der Elektrophorese ist erhöht $(2,4,5,7,10,12)$.

Dtsch. med. Wschr. 117 (1992), 974-977

(c) Georg Thieme Verlag Stuttgart · New York
Pathogenetisch werden Autoantikörper gegen Elastinbestandteile der Lamina elastica muskulärer Arterien angenommen. Auch eine infektiöse bzw. infektallergische Genese wird vermutet. Wir berichten über eine Patientin, bei der lediglich die Femoralarterien von der Riesenzellarteriitis betroffen waren.

\section{Kasuistik}

Anamnese. Im März 1988 stellte sich die 48jährige Patientin wegen ziehender Gesäß- und Oberschenkelschmerzen vor, die vor allem durch Bewegung auslösbar waren. Gelegentlich bestanden auch nachts heftige Schmerzen im Bereich der Hüften und der unteren Lendenwirbelsäule. Unter dem Verdacht auf eine Lumboischialgie waren auswärts bereits krankengymnastische Übungen und nicht-steroidale 
Antirheumatika verordnet worden; die Beschwerden hatten sich jedoch nicht gebessert. Nach einigen Monaten waren die Schmerzen so ausgeprägt, daß die Patientin sich kaum noch bewegen konnte. Zusätzlich bestanden auch ziehende Schmerzen im Bereich der Halswirbelsäule, des Schultergürtels und der Oberarme. Mitte Februar 1989 bemerkte sie zudem krampfartige Schmerzen in beiden Waden, die nach einer Gehstrecke zwischen 100 und $200 \mathrm{~m}$ auftraten und nach kurzem Stehenbleiben verschwanden. Die weitere Vorgeschichte war bis auf eine Appendektomie vor 30 Jahren und gelegentlich auftretende Verspannungen im Nacken bei vermehrter Schreibtischarbeit unauffällig. Kurz vor Beginn der geschilderten Symptome war eine Impfung gegen Frühsommer-Meningoenzephalitis erfolgt.

Körperlicher Befund. Die Patientin befand sich in gutem Allgemein- und Ernährungszustand $(162 \mathrm{~cm}$ groß, $66 \mathrm{~kg}$ schwer). Blutdruck und Pulsfrequenz waren normal. Die Zehen waren kalt und bläulich verfärbt. Die beiden Femoralarterienpulse waren nur schwach tastbar, die Pulse der Arteriae popliteae und die peripheren Fußpulse fehlten. Über der A. femoralis communis beiderseits war ein leises Strömungsgeräusch zu auskultieren.

Klinisch-chemische Befunde. Die Blutsenkungsgeschwindigkeit lag bei $35-43 \mathrm{~mm}$ in der 1. Stunde. Die $\alpha_{2}$-Globulin-Fraktion betrug $12,3 \%$. Das C-reaktive Protein lag mit $4,8 \mathrm{mg} / \mathrm{l}$ noch im Normbereich. Die IgM-Konzentration war auf $610 \mathrm{mg} / \mathrm{dl}$ erhöht, diejenige des IgG betrug 1390 $\mathrm{mg} / \mathrm{dl}$. Der Eisenspiegel befand sich mit $50 \mu \mathrm{g} / \mathrm{dl}$ im unteren Normbereich; die übrigen Routineparameter (Blutbild, Serumelektrolyte, Transaminasen und Urinstatus) waren unauffällig. Die schwach aviden DNS-Antikörper zeigten eine Bindung von $97 \%$. Cardiolipin-Antikörper konnten nicht nachgewiesen werden; die Reaktion auf Lupus-Antikoagulans war schwach positiv

Apparative Verfahren. Dopplersonographisch zeigte sich eine Druckreduktion in den Fußarterien auf $60 \mathrm{~mm} \mathrm{Hg}$ bei einem Systemdruck von $160 \mathrm{~mm} \mathrm{Hg}$ systolisch. Das Hämotachygramm der Arteria femoralis und Arteria poplitea ließ beiderseits einen Verlust der diastolischen Rückflußkomponente erkennen. Die Dopplersonographie der hirnversorgenden Arterien erbrachte unauffällige Befunde. Die Duplexsonographie der extrakraniellen hirnversorgenden Gefäße zeigte normale Gefäße ohne Wandverkalkungen oder Plaques. Angiographisch konnte ein symmetrischer Verschluß der Arteria femoralis superficialis am Abgang sowie ein Verschluß der Arteria profunda femoris am Abgang dargestellt werden. Die A. poplitea füllte sich über Kollateralen, die selbst zum Teil stenosiert waren. Typische arteriosklerotische Wandveränderungen zeigten sich nicht (Abbildung 1).

Therapie und Verlauf. Therapeutisch wurde der Versuch einer systemischen Lyse (9 Millionen I.E. Streptokinase über $6 \mathrm{~h} / \mathrm{d}$ über 3 Tage) unternommen, der jedoch ohne Erfolg blieb. Unter dem dringenden Verdacht auf eine Vaskulitis wurde eine Gewebeprobe aus einer verschlossenen Arteria femoralis superficialis entnommen. Histologisch zeigte sich eine floride Arteriitis, teilweise vom Typ der Riesenzellarteriitis mit Zerstörung der elastischen Strukturen. Schnitte der angrenzenden Muskulatur waren völlig unauffällig, entzündliche Infiltrate wurden nicht nachgewiesen. Die ebenfalls durchgeführte Biopsie der A. temporalis erbrachte dagegen normales Arteriengewebe.

Die Patientin erhielt zunächst für 4 Wochen $60 \mathrm{mg} / \mathrm{d}$ Methylprednisolon. Begleitend nahm sie $100 \mathrm{mg} / \mathrm{d}$ Acetylsalicylsäure ein und betrieb intensives Gehtraining.

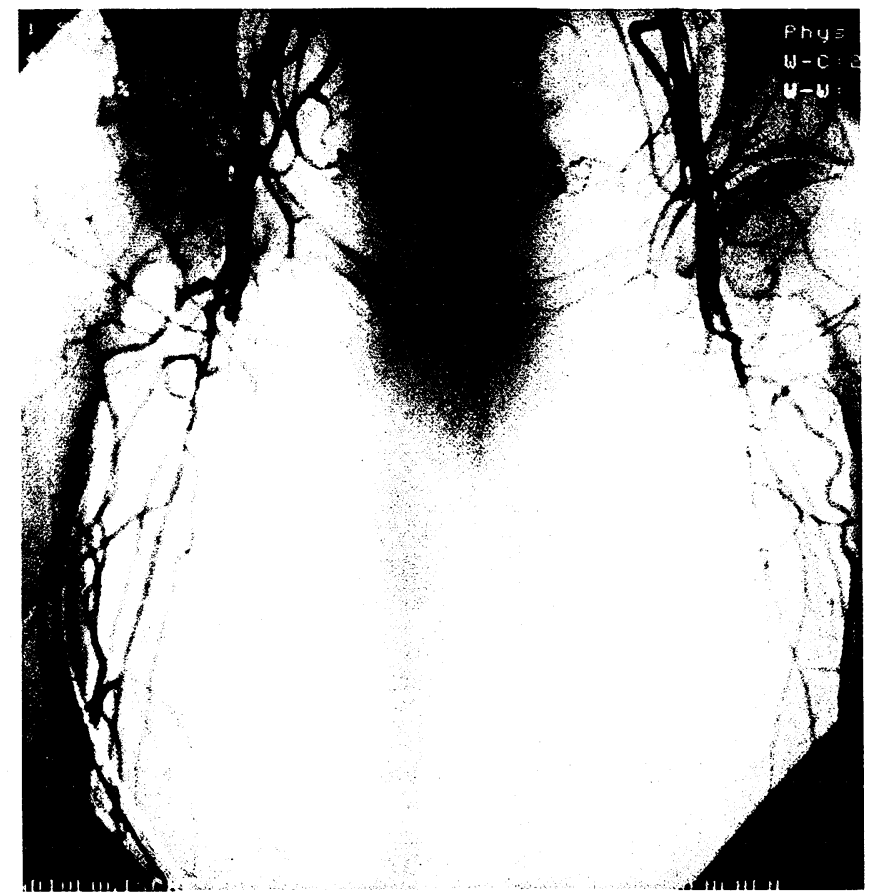

Abb. 1 Digitale Subtraktionsangiographie: Verschluß der A. femoralis superficialis und $A$. profunda femoris beiderseits mit stenosierten Kollateralen.

Schon innerhalb der ersten 4 Wochen ging die Blutsenkungsgeschwindigkeit zurück, und die übrigen entzündlich veränderten Parameter normalisierten sich (Blutsenkungsgeschwindigkeit $29 \mathrm{~mm} \mathrm{Hg} / 1 . h, \alpha_{2}$-Globulin $6,9 \%$, Serumeisen $27 \mu \mathrm{g} / \mathrm{dl}$, IgM $215 \mathrm{mg} / \mathrm{dl}$ ). Die schmerzfreie Gehstrecke verbesserte sich auf 300 Meter. Die peripheren Doppler-Druckwerte blieben unverändert. Ein halbes Jahr später ließ sich dann eine fast unbegrenzte Wegstrecke erreichen. Als Ausdruck einer wohl ausgeprägten Kollateralisation hatten die peripheren Drücke auf $80 \mathrm{~mm} \mathrm{Hg}$ zugenommen bei einem Systemdruck von $130 \mathrm{~mm} \mathrm{Hg}$. Bei konstant niedriger Blutsenkungsgeschwindigkeit und Normalisierung der übrigen Entzündungsparameter wurde die Dosis der Glucocorticoide stufenweise auf 7,5 mg/d Methylprednisolon verringert und nach einem Jahr abgesetzt. Die Entzündungszeichen stiegen daraufhin nicht wieder an. Die Patientin ist bis heute beschwerdefrei und nicht mehr in ihrer Gehstrecke eingeschränkt. Eine Kontrollangiographie wurde deshalb von der Patientin nicht akzeptiert. Duplexsonographisch zeigten sich die bekannten Verschlüsse der Beinarterien mit sehr guter Kollateralisation.

\section{Diskussion}

Die Riesenzellarteriitis kann sich an verschiedenen Arterien innerhalb des Körpers manifestieren $(4,6,16)$. Es gibt Berichte über das Fehlen peripherer Pulse, über Aortenaneurysmen, eine Beteiligung der supraaortalen und der brachialen Gefäße sowie, weit weniger häufig, der Beingefäße $(7,9,11,12,14,18,23)$. Pathologisch-anatomische Studien konnten zeigen, daß neben der Aorta und den Koronararterien, die Carotiden, die Vertebralarterien, die Axillar- und Brachialarterien, die Mesenterial-, Iliakal-, Femoral- und Poplitealarterien beteiligt sind; der Befall ist gelegentlich asymptomatisch und kommt häufiger vor, als das klinische 
Erscheinungsbild vermuten läßt (12). Bei unserer Patientin hatte die Riesenzellarteriitis die gesamten Arterien der unteren Extremitäten befallen, ohne daß eine Beteiligung der supraaortalen Gefäße sowie der Axillar-und Armgefäße festgestellt werden konnte. Auch das Alter der Patientin mit unter 50 Jahren sowie die vergleichsweise geringe Beschleunigung der Blutsenkungsreaktion, die sicher auch zu einer Verzögerung der Diagnose beigetragen hat, werden nicht oft beschrieben $(1,2,4,5,18,23)$. Typische Symptome der Polymyalgia rheumatica bestanden bereits ein Jahr vor der Diagnose. In den letzten Jahren wurde auch über Patienten mit Blutsenkungsgeschwindigkeiten zwischen 30 und $50 \mathrm{~mm}$ in der 1 . Stunde berichtet $(18,24)$. Offenbar muß die Polymyalgia rheumatica bei typischer Klinik auch bei einer nur leicht erhöhten oder normalen Blutsenkungsgeschwindigkeit diskutiert werden $(18,24)$.

Gefäßverschlüsse oder Gefäßstenosen in Armen und Beinen deuten bei Fehlen arteriosklerotischer Risikofaktoren auf eine entzündliche Gefäßerkrankung hin. Bei klinischem Hinweis auf eine Arteriitis temporalis oder Polymyalgia rheumatica mit Muskelschmerzen und Beschleunigung der Blutsenkungsgeschwindigkeit, erniedrigtem Eisenspiegel und $\alpha_{2}$-Globulin-Erhöhung gilt die Biopsie der Arteria temporalis als diagnostische Methode der Wahl, die jedoch nur in $40-50 \%$ der Fälle positiv ausfällt $(3,8,9)$. Wäre sie als einzige diagnostische Maßnahme durchgeführt worden, hätte dies bei unserer Patientin die Diagnose sicher verzögert. Besonders bei Fehlen von Lokalsymptomen ist die Temporalisbiopsie häufig negativ. Deshalb ist es von großer Bedeutung, wenn möglich eine Gewebeprobe aus den betroffenen Arterien zu entnehmen. Der histologische Befund einer Biopsie der verschlossenen Arteria femoralis superficialis erbrachte in unserem Fall den Nachweis von Riesenzellen in der Intima, Media und Elastica interna. Daneben können in einem Gefäß gleichzeitig auch unspezifische Infiltrate der Arterienwände durch Leukozyten und Intimafibrose auftreten, die in einer uniformen Reaktion des Gefäßes zur Stenose bzw. zum Verschluß des Lumens führen können $(6,9,11)$.

Mit bildgebenden Verfahren können bei Fehlen von Plaques und Ulzerationen sowie bei insgesamt glatter Gefäßwand entzündliche Veränderungen von arteriosklerotischen unterschieden wer$\operatorname{den}(11,13,15,16,21,22)$.

Claudicatio intermittens und RaynaudSymptomatik können auf eine weitere vaskuläre Beteiligung hindeuten. Eine Beteiligung anderer als der supraaortalen Gefäße soll in etwa $10 \%$ der Fälle vorkommen. Selten (3\%) sind nur die Beinarterien allein befallen $(6,9)$. Häufiger betroffen sind die Arterien der oberen Extremitäten, gelegentlich auch die Nierenarterien, oder es besteht ein kombinierter Gefäßbefall $(4,7,9,12,13,14,17)$. Die
Therapie der Wahl ist die Gabe von hochdosierten Steroiden (8). Damit kann eine Progression der Erkrankung gestoppt werden. Bei unserer Patientin konnten die Gefäßverschlüsse durch systemische Lyse nicht revaskularisiert werden. Ein operativer Eingriff erschien angesichts der noch tolerablen Gehstrecke und der Tendenz zu Re-Verschlüssen bei Vaskulitis nicht angebracht. Unter Corticosteroidtherapie gingen das Krankheitsgefühl und die Entzündungszeichen rasch zurück; eine Progression der Gefäßveränderungen konnte aufgehalten werden. Dies zeigte sich vor allem in einem deutlichen Anstieg der Doppler-Druckwerte der Fußarterien und der Gehstrecke, die bis zum heutigen Zeitpunkt als unbegrenzt angesehen werden kann. Das ist zum einen auf die medikamentöse Behandlung und zum anderen auf das intensive Gehtraining der Patientin zurückzuführen.

Wir danken Prof. Dr. D. Hahn, Radiologische Poliklinik der Universität München, für die Überlassung der digitalen Subtraktionsangiogramme und Prof. Dr. M. Eder, ehemaliger Vorstand des Pathologischen Instituts der Universität München, für die histologische Befundung. 


\section{Literatur}

1 Biller, J., J. Asconapé, M. E. Weinblatt, J. F. Toole: Temporal arteritis associated with normal sedimentation rate. J. Amer. med. Ass. 247 (1982), 486.

2 Brückle, W.: Die Polymyalgia rheumatica. Internist (Berl.) 28 (1987), 639.

3 Case Records of the Massachusetts General Hospital: Case 351986. New Engl. J. Med. 315 (1986), 631.

4 Chuang, T.-Y., G. G. Hunder, D. M. Ilstrup, L. T. Kurland: Polymyalgia rheumatica. A 10 -year epidemiologic and clinical study. Ann. intern. Med. 97 (1982), 672.

5 Dunker, P. J., J. Ebel: Diagnostik der Riesenzellarteriitis. Dtsch. med. Wschr. 107 (1982), 987.

6 Greene, G. M., D. Lain, R. M. Sherwin, H. E. Wilson, B. M. Mc Manus: Giant cell arteritis of the legs: Clinical isolation of severe disease with gangrene and amputations. Amer. J. Med. 81 (1986), 727.

7 Günther, R., H. Tischler, M. Herold: Polymyalgia rheumatica Klinische Beobachtungen an 90 Patienten. Z. Rheumatol. 44 (1985), 218.

8 Hall, S., J. T. Lie, L. T. Kurland, S. Persellin, P. C. O'Brien, G. G. Hunder: The therapeutic impact of temporal artery biopsy. Lancet 1983/II, 1217.

9 Hamrin, B., N. Jonsson, T. Landberg: Involvement of large vessels in polymyalgia arteritica. Lancet 1965/I, 1193.

10 Healey, L. A., K. R. Wilske: Presentation of occult giant cell arteritis. Arthr. and Rheum. 23 (1980), 641.

11 Hunder, G. G., C. J. Michet: Giant cell arteritis and polymyalgia rheumatica. Clin. rheum. Dis. 11 (1985), 471.

12 Huston, K. A., G. G. Hunder, J. T. Lie, R. H. Kennedy, L. R. Elveback: Temporal arteritis. A 25-year epidemiologic, clinical, and pathologic study. Ann. intern. Med. 88 (1978),162.

13 Jecht, M., W. Klein, K. Howald: Riesenzellarteriitis mit Stenose der A. axillaris beiderseits. Dtsch. med. Wschr. 112 (1987), 119

14 Klein, R. G., G. G. Hunder, A. W. Stanson, S. G. Sheps: Large artery involvement in giant cell (temporal) arteritis. Ann. intern. Med. 83 (1975), 806.

15 Krause, F.-J., C. H. Gleiter: Wert der Angiographie zur Diagnose der Riesenzellarteriitis. Radiologe 26 (1986), 542

16 Overbeck, P.: Riesenzell-Arteriitis der A. axillaris. Dtsch. med. Wschr. 107 (1982), 1453.

17 Perruquet, J. L., D. E. Davis, T. M. Harrington: Aortic arch arteritis in the elderly. An important manifestation of giant cell arteritis. Arch. intern. Med. 146 (1986), 289.

18 Rau, G., F. A. Spengel, H. Dörfler, Ch. Keller, G. Küffer, N. Zöllner: Riesenzellarteriitis mit Befall der Extremitätenarterien. Internist (Berl.) 30 (1989), 622.

19 Ringsgwandl, G., W. v. Scheidt, B. E. Strauer: 61jährige Patientin mit Fieber, Gewichtsverlust, Schmerzen und Sturzsenkung. Internist (Berl.) 26 (1985), 592.

20 Sheehan, N. J.: Alkaline phosphatase and gammaglutamyltransferase in polymyalgia rheumatica and giant cell arteritis. Ann. rheum. Dis. 44 (1985), 644.

21 Stanson, A. W., R. G. Klein, G. G. Hunder: Extrakranial angiographic findings in giant cell (temporal) arteritis. Amer. J. Roentgenol. 27 (1976), 957.

22 Svendler, C. A., S. Söderlundh: Angiographic diagnosis in polymyalgia arteriitica. Acta radiol. Diagn. 18 (1970), 333.

23 Tarnoff, J. F., E. Pearlman, M. H. Balkoura, H. Verstkatumaran: Bilateral superficial femoral giant cell arteritis. J. cardiovasc. Surg. 31 (1990), 375.

24 Wong, R. L., J. H. Korn: Temporal arteritis without an elevated erythrocyte sedimentation rate. Amer. J. Med. 80 (1986), 959.
Dr. Claudia Stautner-Brückmann, Dr. Claudia Haschka,

Dr. J. Bogner, Prof. Dr. F. A. Spengel

Medizinische Poliklinik

der Universität

Pettenkoferstr. 8a

W-8000 München 2

\section{Prof. Dr. G. Baumann}

Gefäßchirurgische Abteilung

Städtisches Krankenhaus Schwabing

Kölner Platz 1

W-8000 München 40 